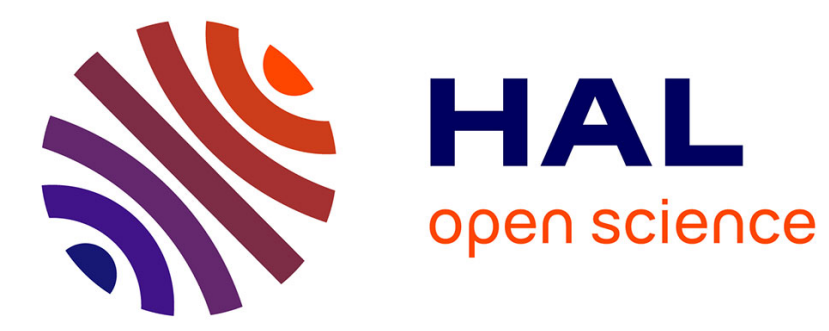

\title{
A Tool to Describe Particle System Evolution from Swarm Robotics Behavior
}

\author{
Ramiro Dell'Erba
}

\section{To cite this version:}

Ramiro Dell'Erba. A Tool to Describe Particle System Evolution from Swarm Robotics Behavior. Advanced Structured Materials, 2020, pp.191 - 217. 10.1007/978-3-030-50460-1_13 . hal-03119197

\section{HAL Id: hal-03119197 \\ https://hal.science/hal-03119197}

Submitted on 23 Jan 2021

HAL is a multi-disciplinary open access archive for the deposit and dissemination of scientific research documents, whether they are published or not. The documents may come from teaching and research institutions in France or abroad, or from public or private research centers.
L'archive ouverte pluridisciplinaire HAL, est destinée au dépôt et à la diffusion de documents scientifiques de niveau recherche, publiés ou non, émanant des établissements d'enseignement et de recherche français ou étrangers, des laboratoires publics ou privés. 


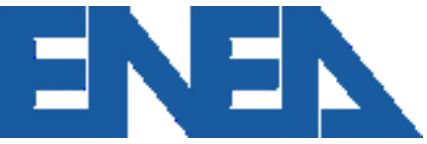

Italian National Agency for New Technologies, Energy and

Sustainable Economic Development

http://www.enea.it/en

http://robotica.casaccia.enea.it/index.php?lang=en

This paper is a pre-print. The final paper is available on:

dell'Erba R. (2020) “A Tool to Describe Particle System

Evolution from Swarm Robotics Behavior." In: Abali B., Giorgio I.

(eds) Developments and Novel Approaches in Nonlinear Solid Body

Mechanics. Advanced Structured Materials, vol 130. Springer, Cham.

https://doi.org/10.1007/978-3-030-50460-1_13 


\title{
A tool to describe particle system evolution from swarm robotics behaviour
}

\author{
Ramiro dell'Erba \\ ENEA Technical Unit technologies for energy and industry - Robotics Laboratory
}

\begin{abstract}
In previous works we have described time evolution of a two-dimensional particles lattice, subject to deformation, without to use Newton's laws. Our experience, in evolution and control of robotic swarm, has suggested as the new position of a particle could be determined by the spatial position of its neighbours; therefore we have used an interaction law based on the spatial position between the particles themselves without defining forces. The tool that we have realized reproduced some behaviour of deformable bodies both according to the standard Cauchy model and second gradient theory. It gives a plausible simulation of continuum deformation and fracture. It can be useful to describe final and sometime intermediate, configuration of a continuum material under assigned strain of some of its points; the advantages are in saving computational time, with respect to solving classical differential equation. Many aspects have to be still investigated, like the relationships describing the interaction rules between particles and its physical meaning and some results does not sound very good. In this paper we try to focus the job done and what is coming over.
\end{abstract}

\section{Keywords}

Position based dynamics; Swarm robotics; deformable continuum

\section{Introduction}

Position based dynamics (PBD) [1] has been widely used in computer animation due to its efficiency, robustness and simplicity. Aim of the PBD is not to compute physical process but to generate visually plausible simulation results with low computational cost [2], sacrificing some accuracy, with respect of solution of heavy differential equations by finite element methods (FEM). This method does not determine forces and solve differential equations but use a position-based approach, where the new position of a particle is determined by its neighbour's positions and can be easily be used to describe complex objects. Owing to the powerful of GPU this new method becomes popular. Systems control of a robotic swarm are often based, like in Nature fish school, on the same principle [3,4]; in our laboratory we are working on this topic since many years (see Figure 1). Therefore, starting from the flocking rules governing the behaviour of single elements in underwater robotic swarm, to reach an assigned geometric configuration, we have adapted the control algorithm in PBD problems [5-7]. Basically the displacement of a particle (discretized continuum equivalent to a swarm element) is determined by the position of its neighbours. Therefore the compute of new position for a particle set can be considered as a constrained geometrical problem leading to a transformation operator between the matrices representing the particles configuration, $C_{t}$, for a discrete set of time steps $t 1, t 2, \ldots t_{n}$. One of the differences, between our methods and PBD, lies in the use of the velocity of the particles often used in PBD; this, in our opinion, hides the dynamic inside so, up to now we avoid using them. So far we tried to describe the deformation of a 
Continuum medium obtaining a useful tool for complex micro-structures not easily analyzed by Cauchy Continuum theory generating and generating big quantity of experimental data. It is known as Classical Cauchy continua are not able to give accuracy prediction in highly non-homogeneous microstructure; to this aim generalizations have to be introduced, either considering additional degrees of freedom to account for the kinematics at the level of the microstructure, [8-20], or including in the deformation energy density higher gradients of the displacement than the first one [21-33]. The latter is a particularly relevant topic considering the technological interest in developing exotic mechanical metamaterials able to perform targeted tasks [34-41], and therefore the investigation of new and efficient algorithms is of great interest at the moment.

The tool we have proposed can exhibit a rich range of behaviours just changing lattice type and its internal parameters. In this paper we resume the work performed focussing on what still need of investigation. In particular we study a beam under shear load, to stress the importance of the parameter's choices and we outline the differences with the solution of the ordinary differential equations.

\section{Method}

Extended description of the algorithm is reported in [5-7]. The two dimensional continuum body is discretized into a finite number of particles occupying, in their initial configuration, the nodes of a lattice. The kind of lattice is chosen between the five plane Bravais lattices but sometime we use also honey comb lattice. This is the first choice we have to do (Choice 1); changing lattice, and the other choices, we can obtain different results with the same initial conditions.

In this lattice we consider four kinds of particles but it is possible to generalize, to describe other behaviours, owing the modular structure of the algorithm. Moreover the role of the particles can be changed during the deformation of the body.

1. The leaders; their motion is assigned and determine the displacement of the other particles.

2. The followers; their motion is determined by the interaction rule with other particles.

3. The frame; it is introduced so that any particle has the same number of neighbours, to avoid edge effects. The motion of the frame particles is determined by the frame rule (see Figure 2 and 3).

4. The ghost; this particles are introduced to describe fracture mechanism. (see Figure 4)

The displacements of the leaders is assigned so does not need any explanation.

How we determine the displacement of the followers? First we have to choice the neighbours of any particles (Choice 2). Typically we used the firs $n c$ particles, where $n c$ is the coordination number of the lattice. This is the case of first gradient theory; but we can also choice a larger set of neighbours, like the neighbours of the neighbours and this is the second gradient theory case. So far we enlarge the set of points with a supplementary shell and this can be generalized to $n$th order interaction (see Figure 2 and 3). Later we have to choice the interacting rule (Choice 3) between the particles. The rule describes the position of a particle as function of the neighbour's positions. As example we can decide to use the centre of gravity rule where the new $x$ coordinate of the particle $j$ is

$$
x_{j}(t)=\frac{\sum_{k=2}^{\text {all neighbours of } j} x_{k}(t)}{N}
$$

Where $\mathrm{N}$ is the total number of neighbours and similar equation can be used for the $y$ coordinate. By this way displacement of a follower point is the average value of the displacements of its neighbours; the number of shells determine the order of interaction. We can use different rules in order to imitate different constitutive equations. Possible generalizations of Eq.1 are geometric, power and weighted mean. Possible weight is the particles Euclidean distances $\operatorname{dis}(k, j)$ between the particles $k$ and $j$. This can simulate Hook law, where recalling force is increased with increasing deformation. By Eq.1 we note as $x$ and $y$ coordinate are independent so Poisson effect cannot be obtained. A possibility to obtain it is to use

$y_{j}(t)=K *\left(x_{j}(t)-x_{j}\left(t_{0}\right)\right) * d a+\frac{\sum_{k=2}^{\text {all neighbours of } j} y_{k}(t)}{N}$

Where $d a$ is a function of the distance from the central axis, $K$ a parameter determining the response force and $x\left(t_{0}\right)$ the $x$ coordinate at time $t_{0}$. So far expansion of $x$ coordinate has effect on the $y$ coordinate. The Euclidean distance, $\operatorname{dis}(k, j)$, can be used as weight.

$x_{j}(t)=\frac{\sum_{k=2}^{\text {all neighbours of } j} \operatorname{dis}(k, j) x_{k}(t)}{\sum_{k=2}^{\text {all neighbours of } j} \operatorname{dis}(k, j)}$ 
We can also force the follower's movement to overcome the barycentre equilibrium position, leading the lattice to oscillate.

$x_{j}(t)=\frac{\sum_{k=2}^{\text {all neighbours of } j} w(k, j) x_{k}(t)}{\sum_{k=2}^{\text {all neighbours of } j} w(k, j)}+f d\left(\frac{\sum_{k=2}^{\text {all neighbours of } j} w(k, j) x_{k}(t)}{\sum_{k=2}^{\text {all neighbours of } j} w(k, j)}-M T\left(j, t_{0}\right)\right)$

Where $w(k, j)$ is the weight, $f d$ is a feedback factor and $M T\left(i, t_{0}\right)$ is the $x$-coordinate of $j$ point at $t_{0}$ to have memory of the initial configuration.

The compute of new position for a particle set can be considered as a constrained geometrical problem using a transformation operator between the matrices describing particles configuration, $C_{t}$, for a discrete set of time steps $t 1, t 2, \ldots . t n \ldots . .$.

In the algorithm, the neighbours can dynamically change at every time step. In this paper we choice to fix the neighbours of every particle at the initial time $t_{0}$, and not to change them during time evolution of the configurations; this has the mean to consider a crystalline lattice and therefore to deal with solid phase materials. The concept of neighbours is Lagrangian, and neighbourhood is preserved during the time evolution of the system; the only exceptions arising with the fracture algorithm, not considered in this paper. Also the definition of neighbours is customizable by changing metric; for example we can consider points whose Euclidean distance (weighted or not is another possibility to take into account anisotropies) is less than a threshold, instead of the coordination number of the lattice.

Starting from the leaders motion each time step the displacement propagates of one shell, determined by the neighbours, up to involve all the particles.

To avoid edge effects we build a frame surrounding the body by an external shell of point, so that any follower interacts with the same number of elements. The motion of the frame is simple: it only follows the motion of an assigned follower of its competence; in case the assigned followers are more (i.e. in a corner) then an average displacement, or a more generic complex rule (Choice 4), is considered.

The ghost points appear in case we want to describe fracture phenomena. To this aim we choose a threshold, $d f$, (fracture distance Choice 5) to be overcome to declare fracture. If the distance between points is larger than this threshold they stop to influence each other so they are no longer taken into account in the calculation of the follower position. To preserve symmetry we introduce ghost points with the purpose of balancing the calculations of the point's displacements, just to balance the equations. Typical position, where we put ghost points (Choice 6 ), is that is able to recover the original shape of the lattice (see Figure 4). Anyway other choices lead to different results [5-7].

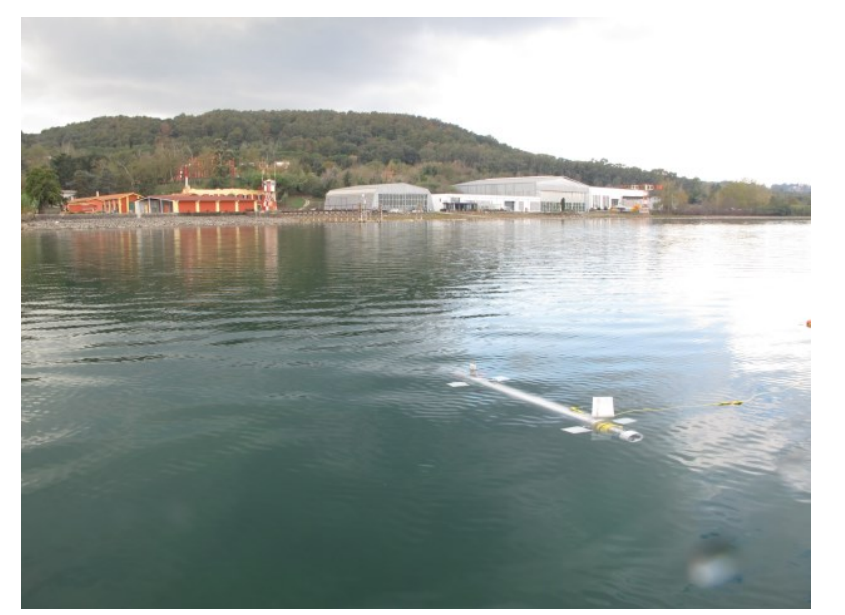

Figure 1 VENUS, element of the swarm realized in our laboratory

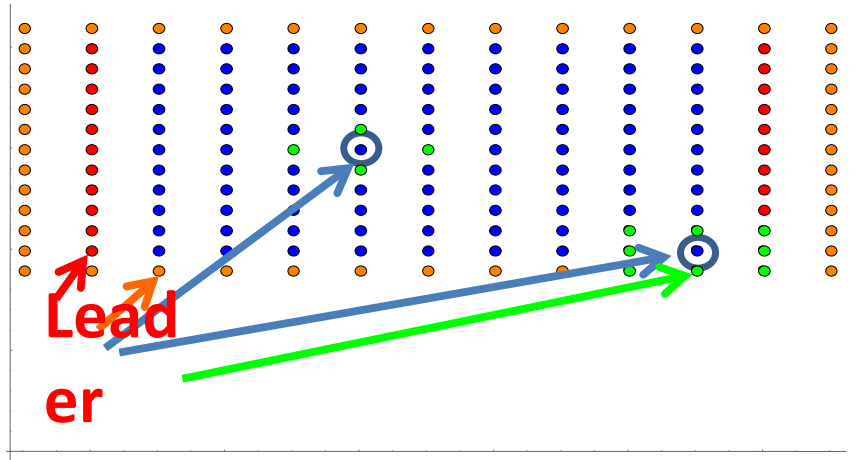

Fra Гi, Ire 2 Kind of particles

\section{Follo}

wer

Neig 


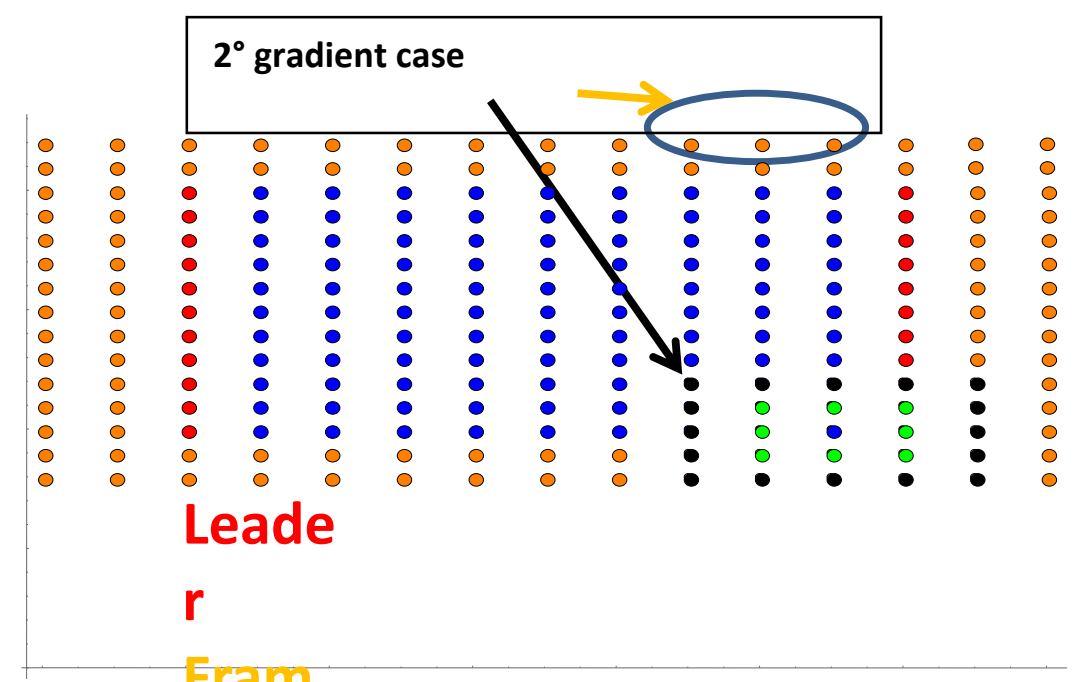

Figura 3 Kind of particles $\left(2^{\circ}\right.$ gradient case)

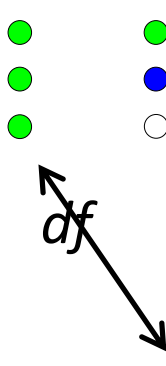

Figura 4 Fracture mechanism: ghost

In the net future we are considering the possibility to discuss the proposed model in a fully variational setting, which is by nofolotrivial but would provide clear methodological advantages (see [42] for an introduction and [43-47] for illustrative cases concerning continua with non-classical properties); we like to introduce pseudoenergevedonsiderations by two formulations PE1 and PE2. The first is the sum, extended to the neighbours, of squares of the differences between the distances of the point from its neighbours minus the distance in the intitie configuration i.e.

$$
\begin{array}{ll}
\text { bours } & P E 1(t, j)= \\
\text { lst }
\end{array} \sum_{k=1}^{\text {all neighbours of } j}\left(\operatorname{dis}(t, k, j)-\operatorname{dis}\left(t_{0} k, j\right)\right)^{2}
$$

Where dis $(t, k, j)$ gh the Euclidean distance between points $k$ and $j$ at time $t$. The reason for this choice lies in the attempt to emblotêpestential energy of material point subject to Hook law.

To compare time contiguous configuration $C_{t}$ and $C_{t-1}$ we define for each point $j$ and each time $t$

$$
\text { 2nd } \quad P E 2(t, j)=\left\|C_{t}-C_{t-1}\right\|
$$

Where $\|$ is the Norm of the vector defined by the point $j$ at time $t$ and $t-1$.

It must be underlined that this artifice has no direct connection with the usual energy definition (this is the reason we use the term pseudoenergy) but could be useful to understand deformation. However in this paper we do not consider PE1 and PE2 function; investigation about them can be found in[5-7].

\section{Results}

In this section we briefly resume some significant results obtained by this tool in the preceding works [5-7] where different strain of the leaders, with different choices, have been investigated. The behaviour of some more complex ASTM sample and the respect of Saint Venant principle have also been described; moreover we are looking for the limits of the tool, so we have found some not satisfactory results that will be discussed in this paper.

\section{Case a) Tensile test}

Consider a square shape specimen subjected to pull and release in tensile test. We are considering a sample undergoing strain from one side (the other side is clamped) at constant velocity in x direction (speed 0.6 unit length/step time), with a square lattice 10x10 unit. At a certain time the pull is released and the leaders return to original configuration (this mean that leaders have changed category and now are followers) attracted by the other points. The simple rule, governing follower's motion is that every point must be placed in the barycentre of its neighbours (Eq. 1); the neighbours are determined by the coordination number of the lattice; therefore the leader's motion implies a displacement of the first layer that propagates in successive time steps to the other particles. This means the displacements, at each time step, involve a larger shell of points until to regards all the lattice points. In second gradient [5-7] we have considered also the neighbours of the first neighbours. In Figure 
5 we can see the configuration of the lattice over different time together with the PE1 contour plot. Red points are the leaders; blue the followers and orange the frame. From the figure we can outline that the $\mathrm{x}$ displacement of the points seems do not depend on the y coordinate; however looking at the PE1 picture we can note a light convexity that does mean this is not true.
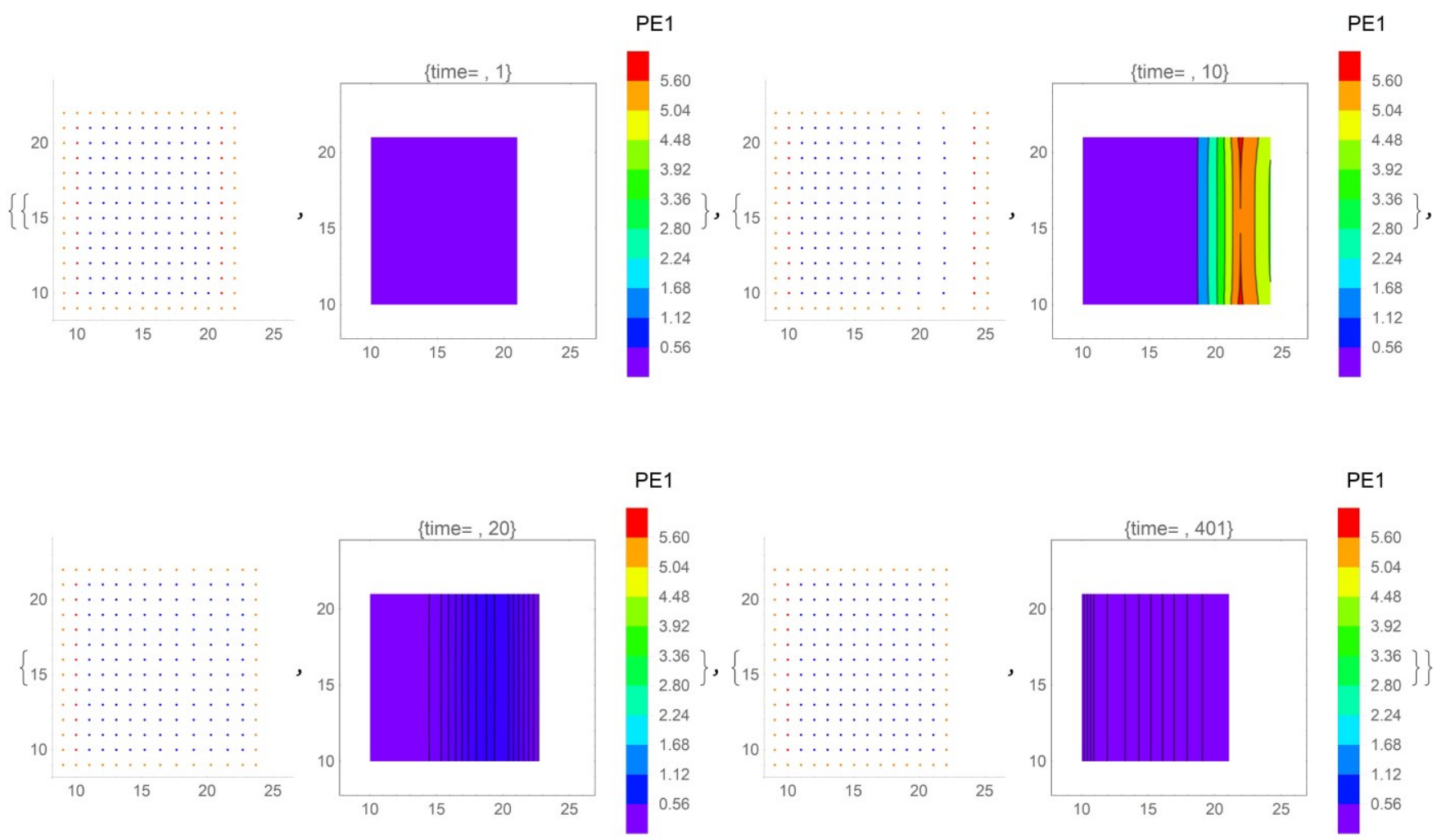

Figure 5 Configuration of the lattice over different time (1,10, 20 and 401) and PE1 contour plot

A deeper examination of the point's displacements confirms as close to the frame the displacements, along $\mathrm{x}$ coordinate, are lower with respect to central points. This can be explained as an edge effect. In fact if we consider points on the same vertical lines those that are close to the frame follow the neighbours with a little delay owing to the different rule determining the displacement of the frame and of the followers. So they see a different situation with respect to a central point. Moreover we can note as the maximum value of PE1 (red area) is not on the leader line but just one line on its left; this because, in this case, the leaders have in their neighbours, some points of the frame that always are close to them. We can avoid this convexity effect using a different frame or mirroring the followers to obtain an infinite sample. Finally it should be noted as at $t=401$ the lattice is not back completely to the reference configuration owing to asymptotic process of relaxation. Consider now a central point $j=67$ (sixth column, seventh row, points are numbered from left to right and from bottom to up) of the lattice. The value of the PE1 increases notably when points are pulled, after a delay owing to the propagation time as can be seen in Figure 6; it decrease when the leaders become followers subjected only to the rules leading to equilibrium barycentre position. If we change point the shape of the curve remains the same but can be less o more flared. In Figure7 and Figure 8 the evolution with time of the coordinates of central point $j=67$ and close to the leaders $(\mathrm{j}=115)$ are shown. Also in this picture we can recognize the coordinate $\mathrm{x}$ increases linearly (velocity is constant), after a delay (less for $\mathrm{j}=115$ ), owing to the propagation time and later decrease to the original position. 


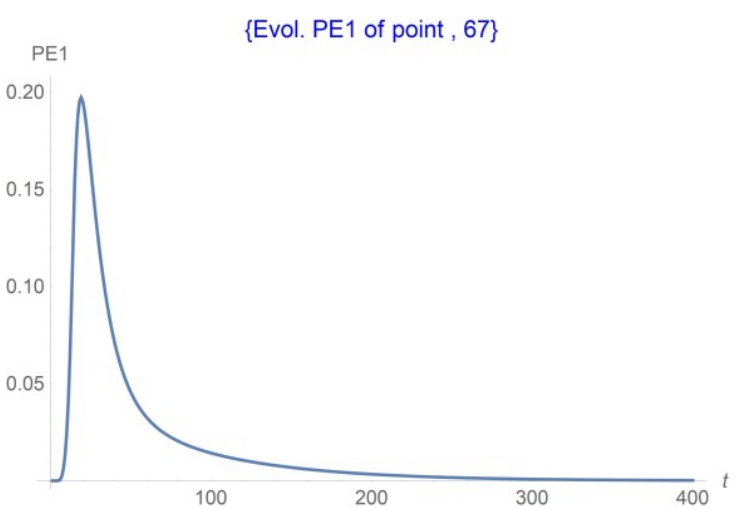

Figure 6 PE1 of the central point's $j=67$ versus time

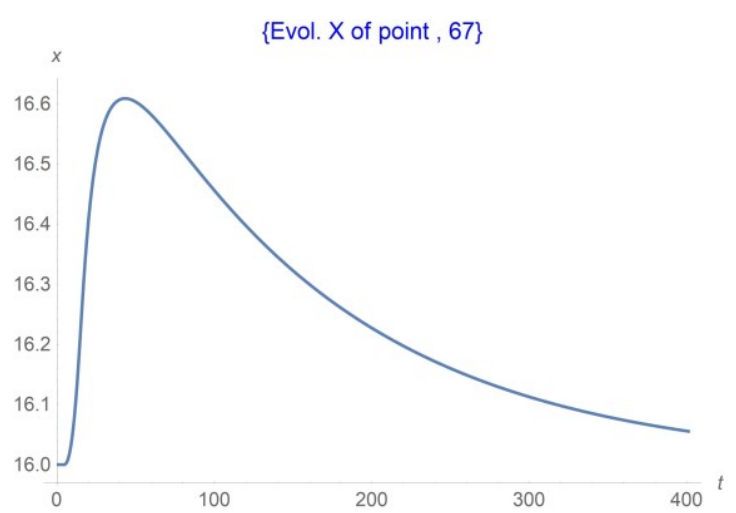

Figure $7 \mathrm{X}$ Evolution of the point $\mathrm{j}=67$ versus time

A light modification can generate instabilities and oscillations; as example we can add to Eq.1 a feedback term proportional to the difference between actual and initial position to overshoot the old equilibrium position. The result is showed in Figure 9 and Figure 10 (we used different feedback)Figure 6; note as also the reaction time is changed. Using an higher feedback instabilities can be generated (see Figure 11).

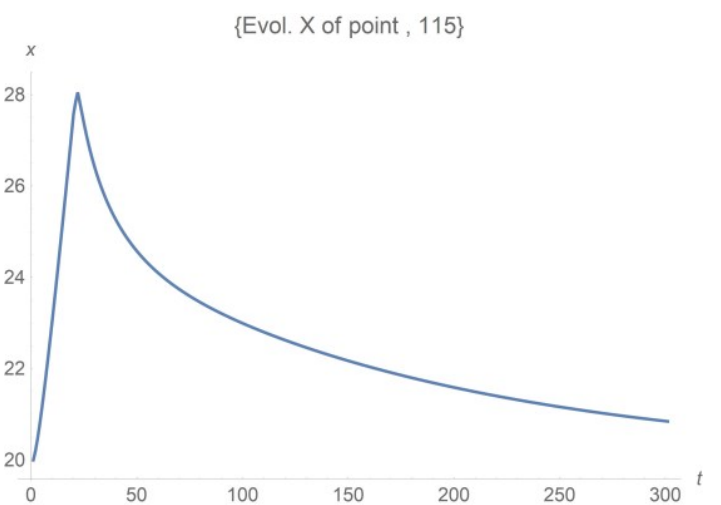

Figure $8 \times$ Evolution of the point $\mathrm{j}=115$ versus time

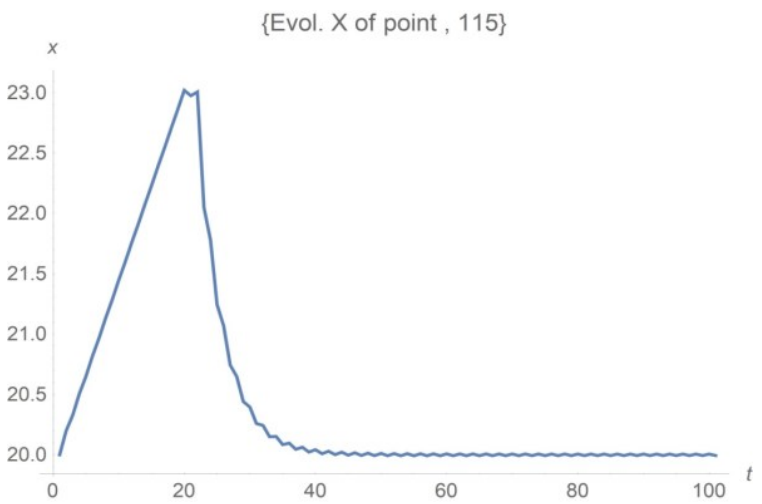

Figure $10 \times$ Evolution $\mathrm{j}=115$ versus time (modified rule with feedback)

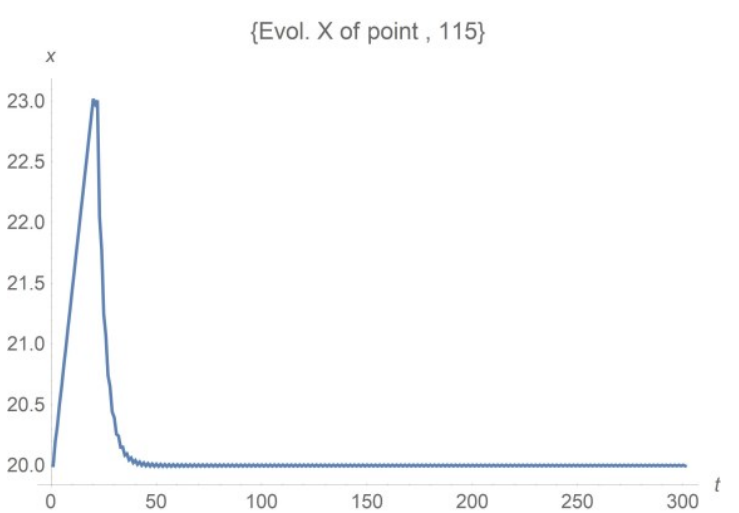

Figure $9 \times$ Evolution $\mathrm{j}=115$ versus time (modified rule with feedback)

$\{$ Evol. X of point, 67\}

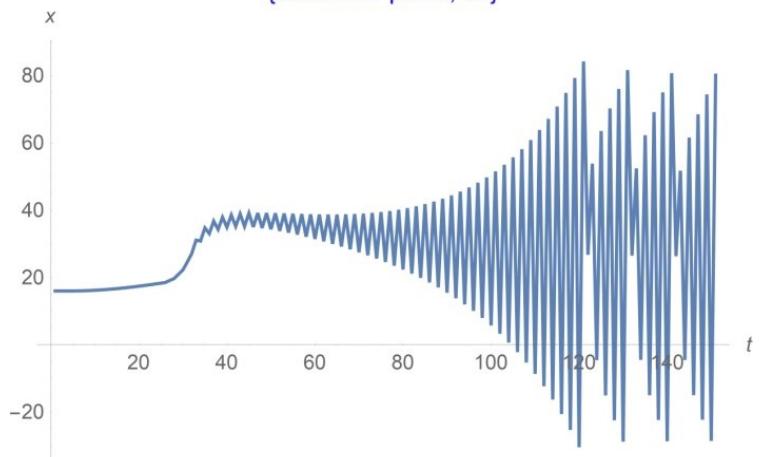

Figure $11 \times$ Evolution of the point $j=67$ versus time (modified rule with feedback)

Case b) Shear test with Poisson effect

An interesting case, always using a square lattice, can be seen in Figure12 were the final configuration is shown after a shear test. Here we have used a rule for the follower making use of "mixed coordinate", which means the $\mathrm{y}$ coordinate is dependent on the evolution of the $\mathrm{x}$ coordinate. This allows us to obtain lateral contraction, i.e. Poisson effect. The result of the shear test is a strange "window" flag. We have used a first and a second gradient model, changing the shell of neighbors. Differences are in a more stiff reaction in the second gradient case, owing to the larger numbers of neighbors involved in calculating the follower's positions. Owing to the lateral contraction, the y coordinate of points above this axis decrease, with shear, while below they are increased. Note 
the delay reaction time, because the involved points have to be informed about the displacement of the leaders.
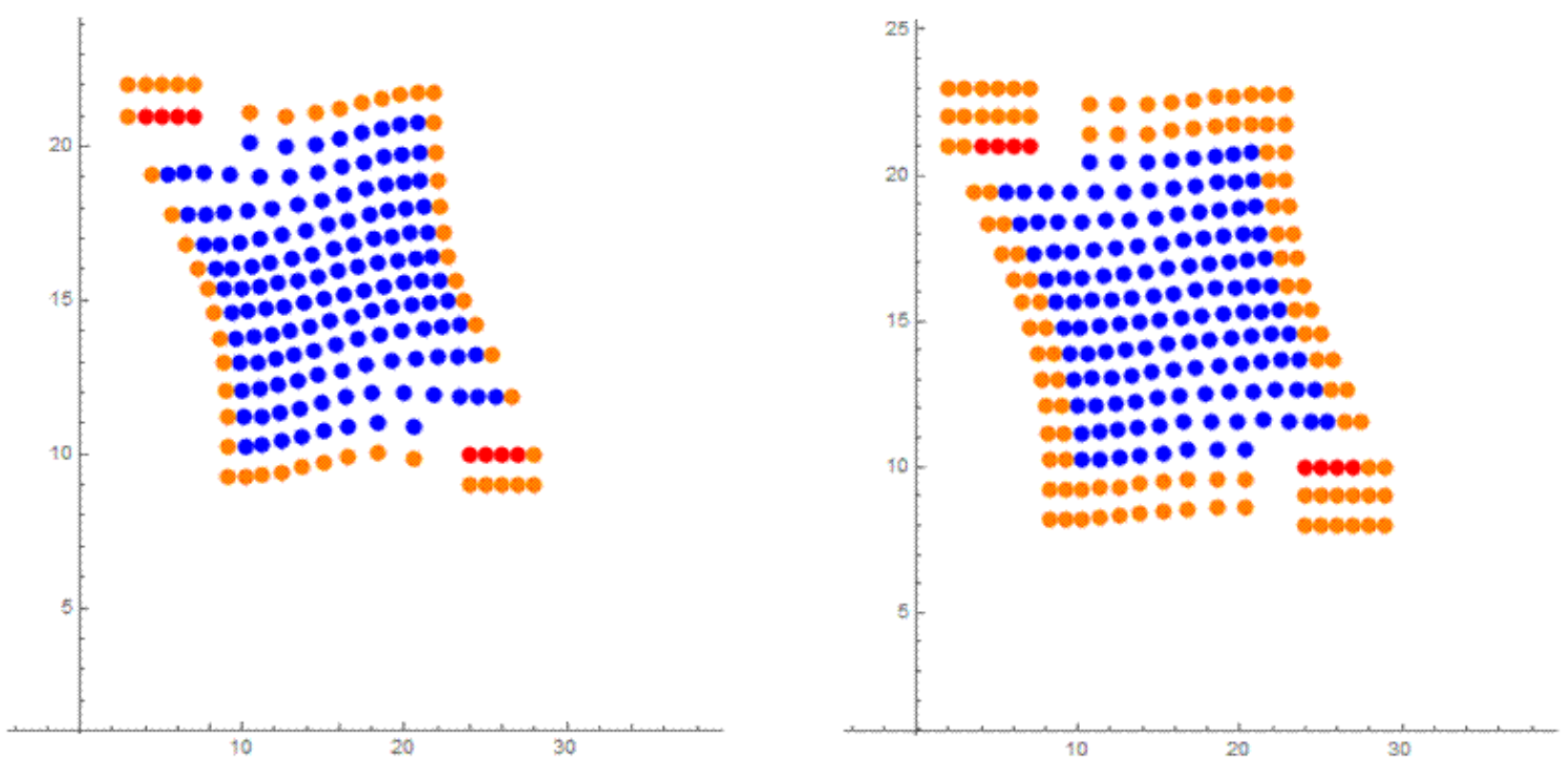

Figure12 Final configuration of the lattice with Poisson effect in shear test square lattice, first and second gradient case.

\section{Case c) Fracture test}

For the next example we shall consider a square sample undergone a tensile test with fracture (Figure 13 just before fracture). Fracture distances are 6 units and speed is 0.5 units/step, for 150 steps long. When a distance between the points is larger than fracture distances the sample is broken and the followers go to equilibrium position; if no followers remain attached to the leaders (it depends on the distances, we shall see later in other cases) they return to their initial position. As explained in a preceding work [5-7] the convexity, in the fracture mechanism, is related to the presence of the frame. Analysis of PE1 plot show as, before fracture, there are areas of stress concentration. Higher stress areas are close to the leaders. The trend of the follower points is quite linear during traction but it becomes non linear when the followers remain alone and return back. This because the traction is imposed with constant speed while the reassembly of the points is driven by the follower's rule. Once again involving a larger number of neighbors lead to a more stiff behavior as can be seen in Figure 14 (second gradient case). 


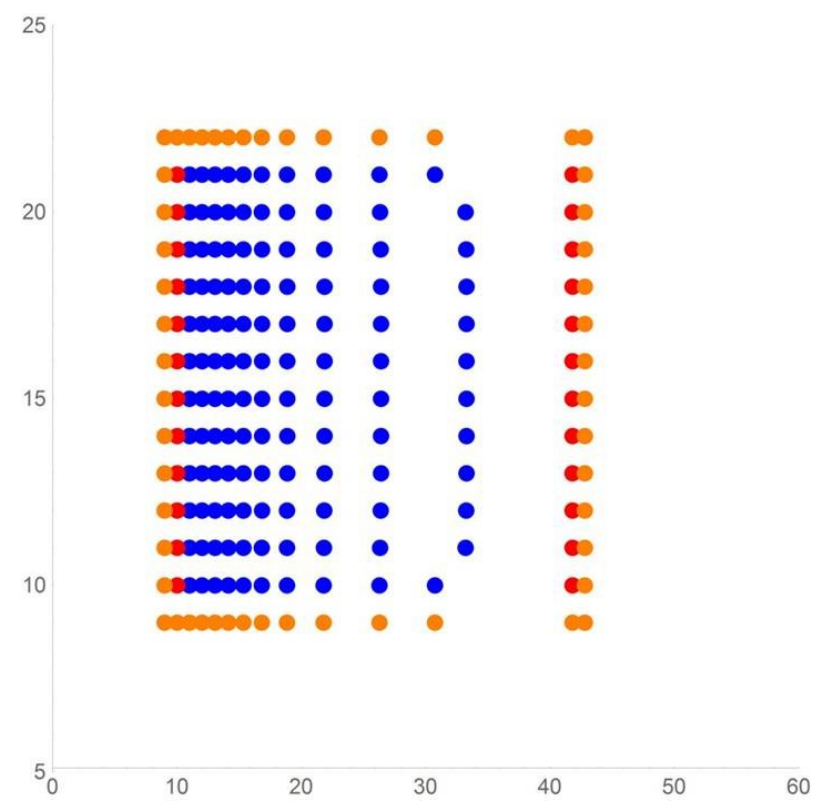

Figura 13 Fracture test of square specimen square lattice

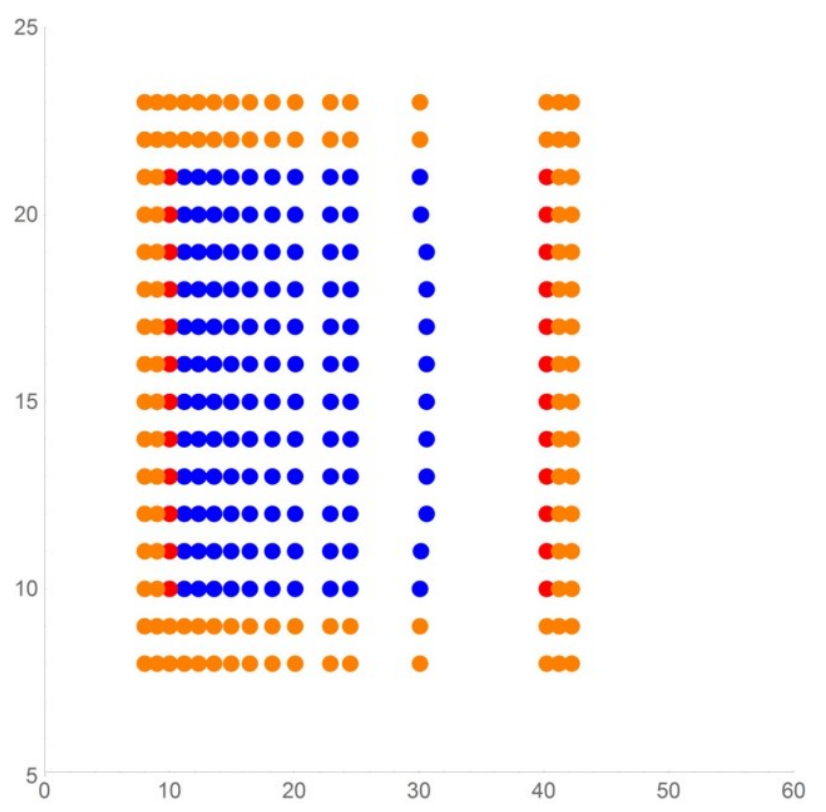

Figura 14 Same as Figure 13 but second gradient

Case d) The importance of the frame rules

In the case of hexagonal lattice we can choice two different rules for the frame, as can be seen from Figura 15. In the first case a frame point displacement is just the same of the corresponding follower. But in some case, like hexagonal lattice, you can decide there are more than one corresponding followers and choice the point frame displacement as the average value of them

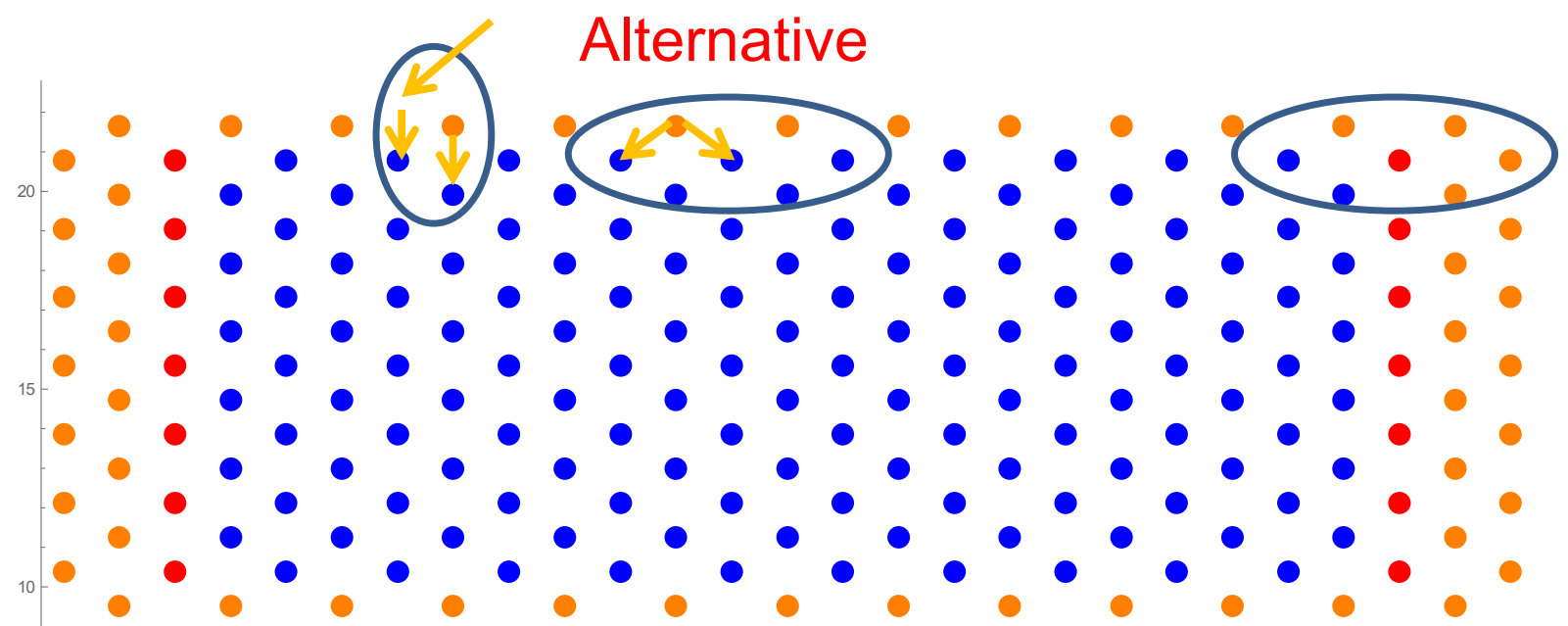

Figura 15 Alternative choice for the frame rule displacement. In the first case each frame point is moving as a corresponding follower. In the second case as the average value of more followers assigned.

This lead to different final deformed configuration as can be outlined in Figure 16 and 17 . The presence of a frame point in the middle leads the final configuration to a concavity. The absence of followers on the right side, once 
again is depending on the leader's speed.

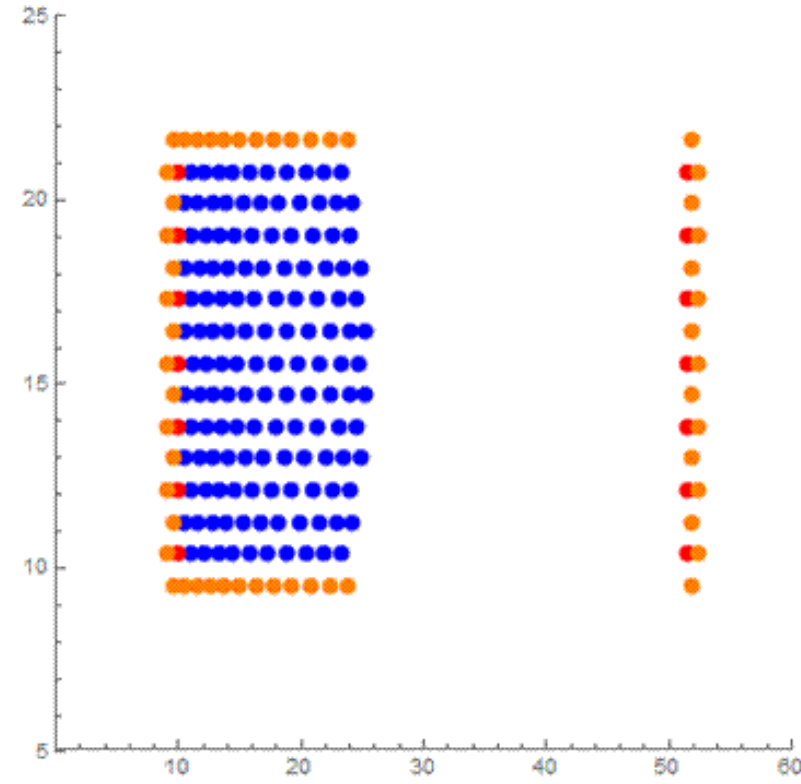

Figura 16 Final configuration in fracture test hexagonal lattice

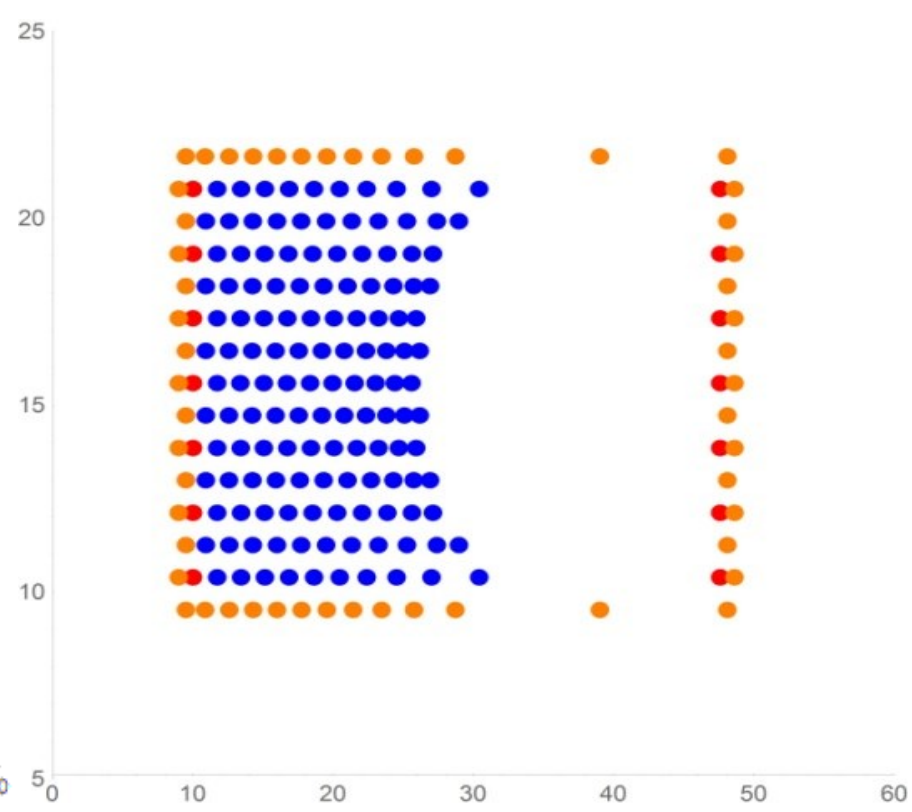

Figura 17 Same as Figure 16 but with different frame rule

Case e) The importance of the lattice

Consider now consider an oblique lattice (Figure 18). Owing to the asymmetry (see look at the five red leaders on the right) of the leaders with respect to the frame a particular breakage fracture can be observed. In fact if we consider a symmetry axes in $\mathrm{x}$ direction we can note two leaders close to the frame in the upper level and only one close to the bottom. This leads to a fracture starting from the bottom where the attraction of the leaders is lower. It seems to rip a piece of paper. The fracture distance is 10 units and the speed is $0.4 \mathrm{unit} /$ time step.

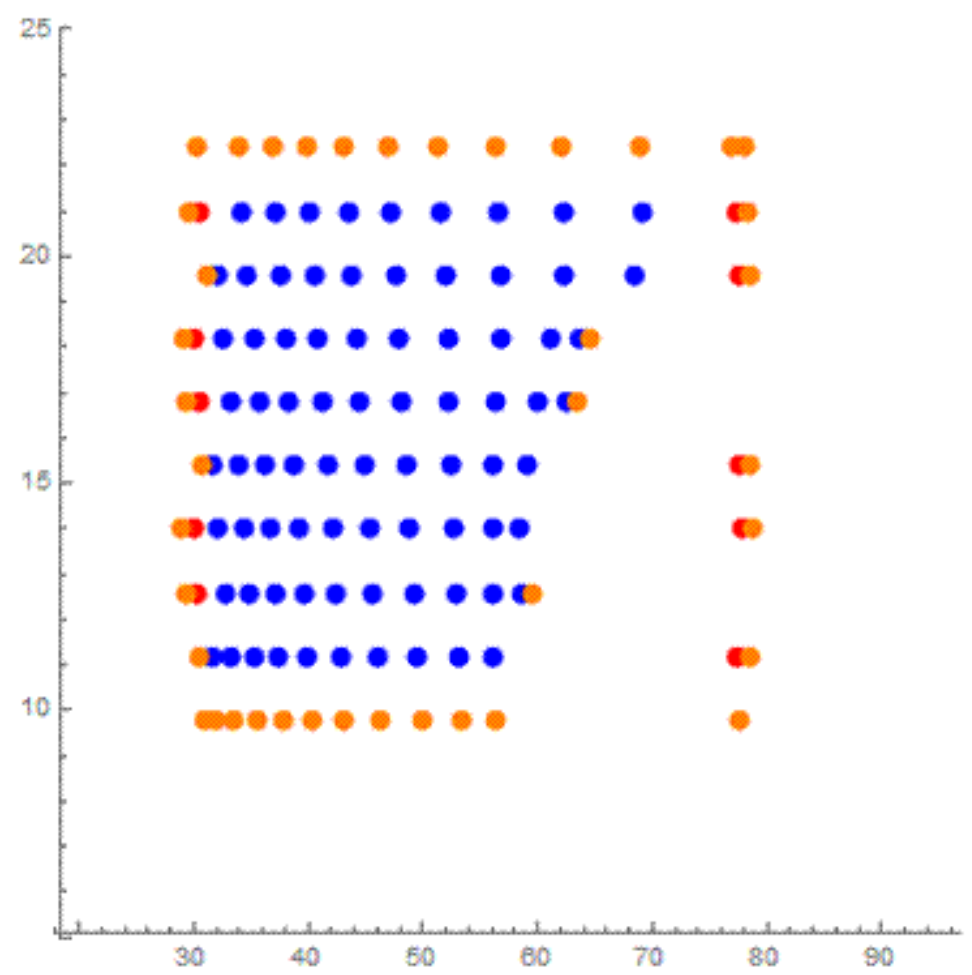

Figura 18 Fracture in tensile test with oblique lattice.

Case f) Fracture the importance of the lattice and of the neighbours 
In this case we use a rectangular centered lattice for tensile test but we reduce the neighbour's number, $n c$, to five. This gives more mobility to the model leading to a more plastic behaviour and increasing the number of the detached points; The fracture mechanism is quite different together as well as the final configuration (see Figure 19). This example shows, once again, that change in model parameters lead to different behaviours.

The lower numbers of particles involved render the sample much more fluid, allowing detachment of a larger number of particles, as we can see on the right side of the pictures (see Figure 20).

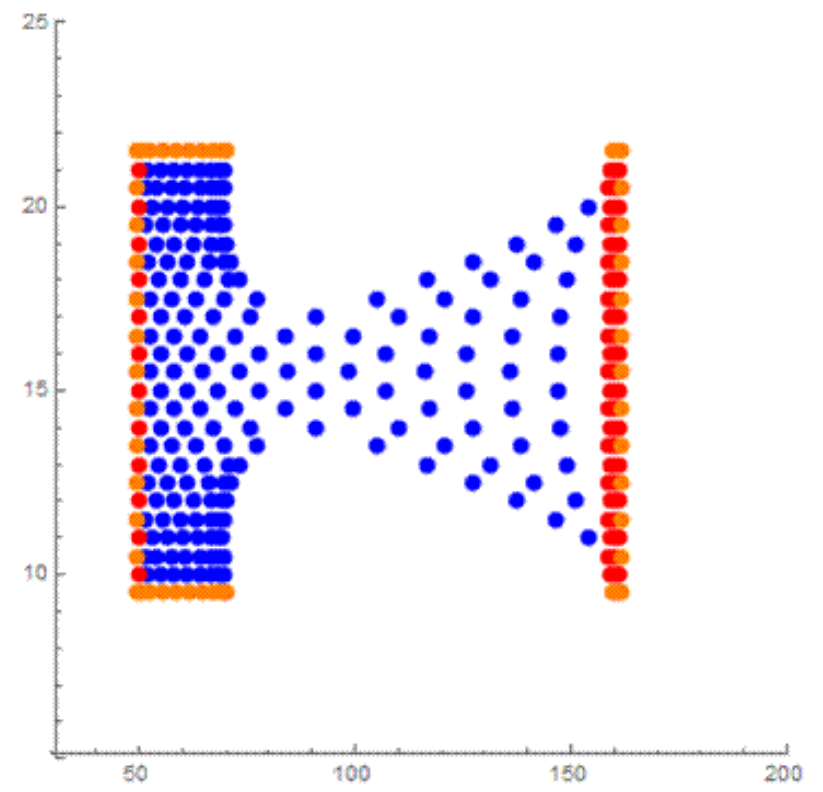

Figura 19 Fracture ion tensile test rectangular centered lattice nc=5

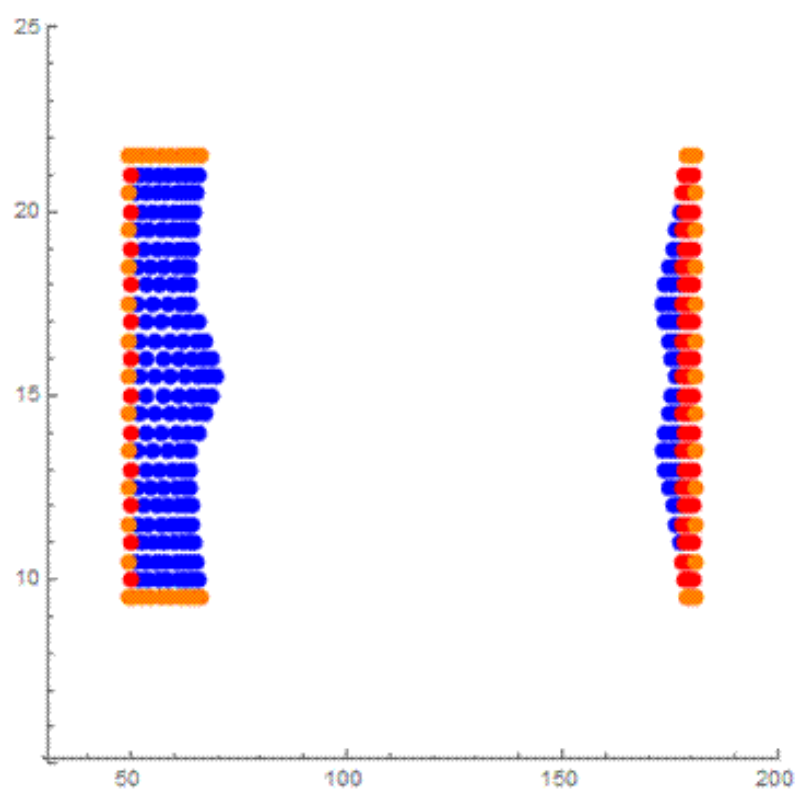

Figura 20 Final configuration of Figure 19

\section{Case g) ASTM test}

In order to investigate sample behavior of more complex shape we consider specimen ASTM D638 standards for tensile tests. The specimen is clamped at both ends on a surface and pulled on one side, so, in this case, the leaders are many; the test speed is of $2.5 \mathrm{unit} / \mathrm{step}$, in x positive direction, for 150 traction step e 2500 relaxation step. We shall consider tensile test with Poisson effect, change of the lattice and fracture; many other cases can be found in the references. In Figura 21 the sample before the test is shown; note the double set of frame points because we are considering $2^{\circ}$ gradient case. A larger number of time steps is required for relaxation, owing to the larger number of points used to describe the specimen; this does not mean a longer relaxation time, because unit time is arbitrary, only because the influence of the displacement propagates at two shell (second gradient case) each time step we need many steps to involve the whole sample. In Figura 22 the elongated sample (first gradient case) whiles in Figura 22 the second gradient case. Once again the second gradient case seems to be stiffer with respect of the first gradient. It should be noted that the sample does not reach a symmetric final configuration as we can expect (see last figure); we have also wait for 10000 time relaxation steps without modification. On the contrary if we use a rectangular shape sample it does (the points are equally spaced). There is no physical reason for this, our opinion is that this effect is linked to the particular equilibrium condition generate by the geometry. We are working on this and on higher gradient computations. In the two cases we are considering the Poisson effect it is possible to see lateral contraction. It seems the points cluster to create islands but this effect must be investigated better. In case of second gradient interaction this does not occur, as can be seen in Figura 23. The fracture test (see Figura 24, Figura 25 and Figura 26) is considered for the rectangular centered lattice. Distance fracture is 11 units and the speed was $0.6 \mathrm{step} /$ unit time. As can be seen, the fracture occurs close to the top of the profile and not in the central area. Studies, in progress, show as the fracture zone can be moved by varying working conditions. We can render the fracture more or less brittle changing the model parameters like neighbors' number, type of lattice, speed etc. As example in second gradient the same sample has a more brittle behavior; or if I use a speed of $2.5 \mathrm{step} /$ unit time in the same condition I will get no followers on the right side of the fractured sample. We can observe differences in the internal distribution on the points and in the convexity of the propagation front of the deformation i.e. see the convexity of the points owing to the different lattice used 
compared to the preceding case.

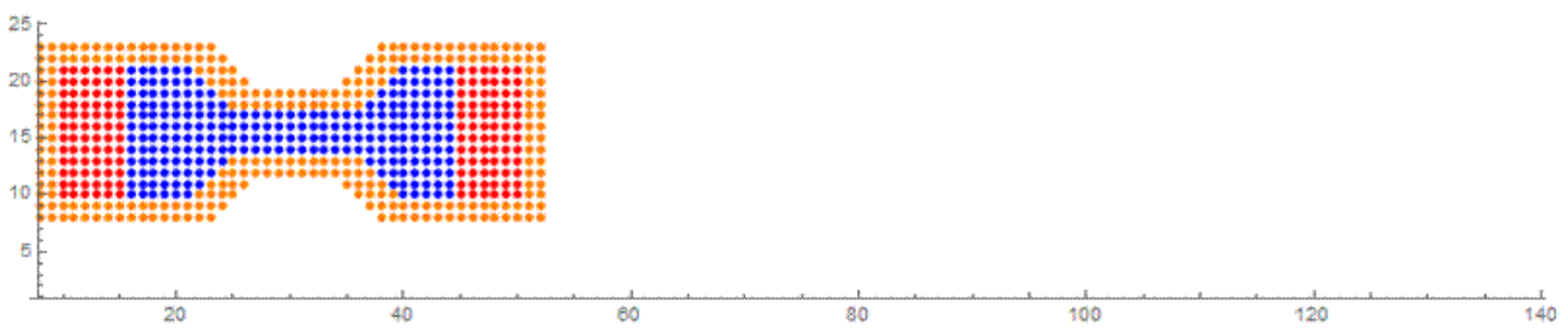

Figura 21 ASTM sample $2^{\circ}$ gradient before tensile tests

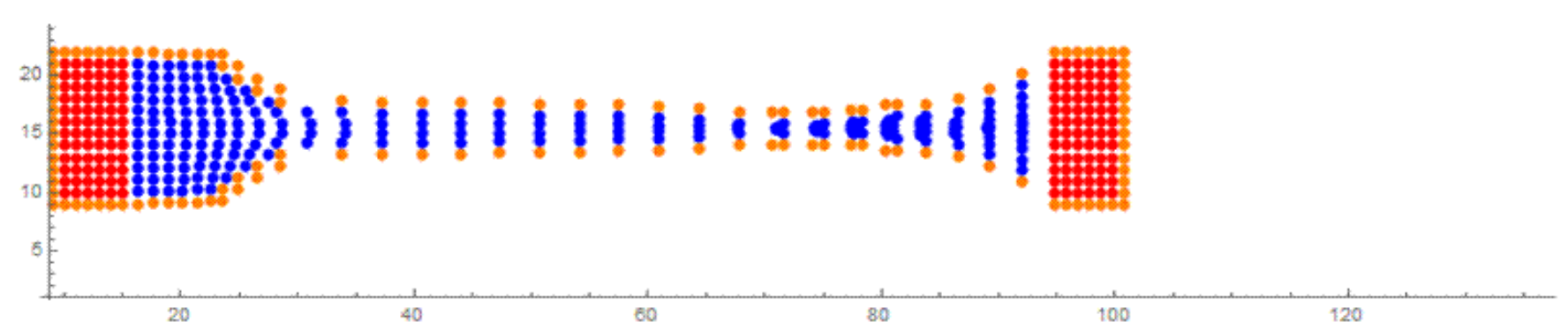

Figura 22 ASTM sample Poisson effect square lattice

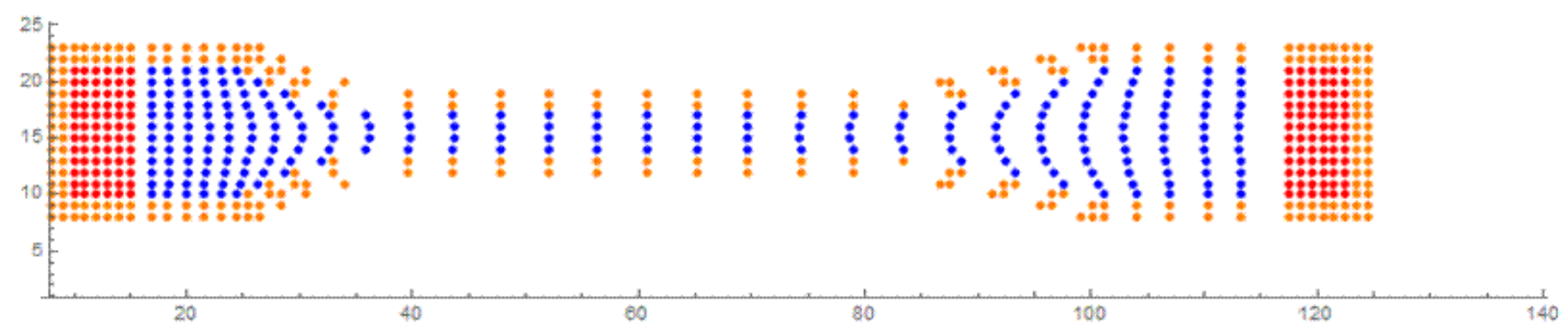

Figura 23 ASTM sample Poisson effect square lattice $2^{\circ}$ gradient

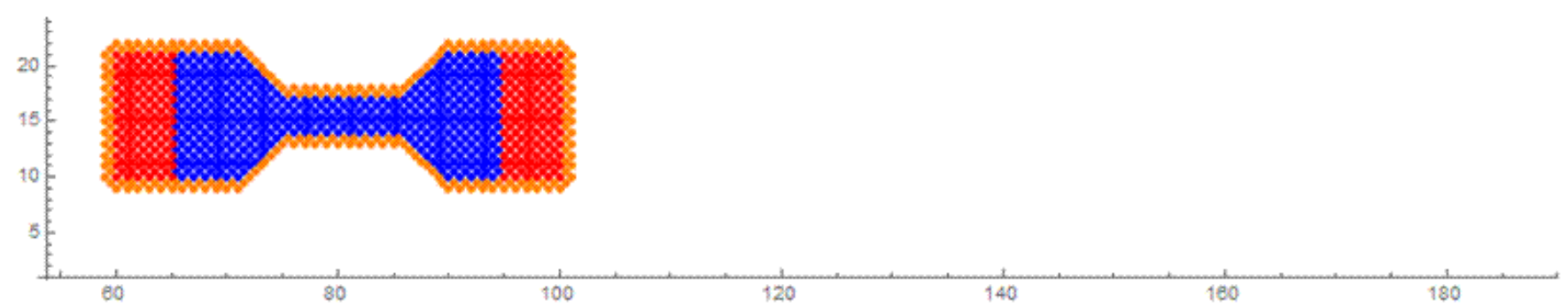

Figura 24 ASTM rectangula centered lattice before tensile test

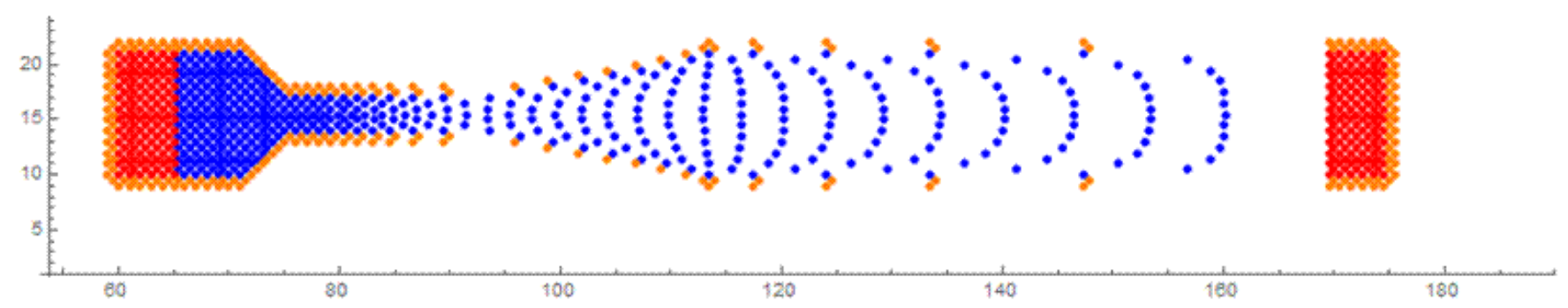

Figura 25 ASTM rectangula centered lattice after tensile test 


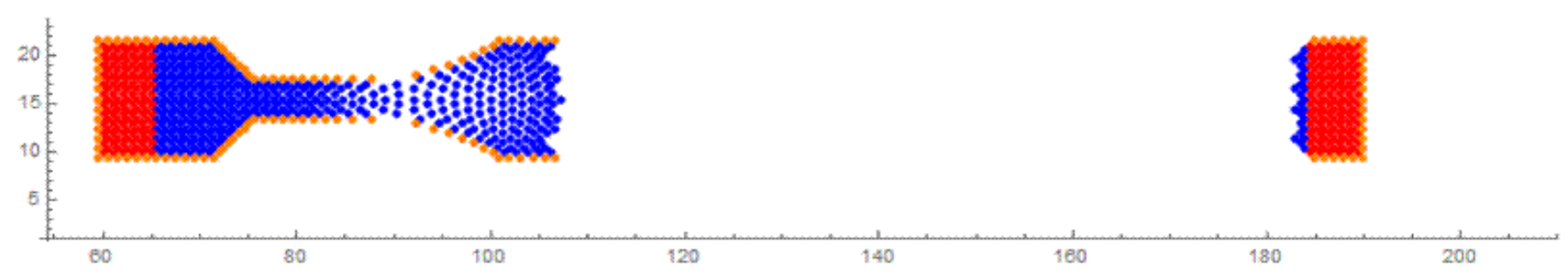

Figura 26 ASTM square lattice fracture

\section{Case h) Short beam}

\section{The beam}

In this section we want to investigate the behaviour of our tool applied to a bending beam loaded at one end and evaluate the differences with the numerical solutions of the differential equations. We consider a bi-dimensional elastic short square beam ( $\mathrm{X}$ and $\mathrm{Y}$ coordinate from 10 to 21 in the not deformed configuration) with materials parameters $Y=1000 \mathrm{~N} /(\mathrm{m} 2)$ and $v=0.33$, where $Y$ is the Young modulus, $v$ the Poisson coefficient; this value are close to that of a medium molecular weight polypropylene [48-50]. Boundary load is on the right (Neumann condition) and no displacements on the left (Dirichelet conditions).

$$
\begin{aligned}
& \frac{\mathrm{Y}}{2(1+v)} \nabla^{2} \mathbf{u}+\frac{\mathrm{Y}}{2(1-v)}\left(\frac{\partial}{\partial x} \mathbf{u}+\frac{\partial}{\partial y} \mathbf{v}\right)=0 \\
& \frac{\mathrm{Y}}{2(1+v)} \nabla^{2} \mathbf{v}+\frac{\mathrm{Y}}{2(1-v)}\left(\frac{\partial}{\partial x} \mathbf{u}+\frac{\partial}{\partial y} \mathbf{v}\right)=0
\end{aligned}
$$

$\mathbf{u}(\mathrm{x}, \mathrm{y})$ and $\mathbf{v}(\mathrm{x}, \mathrm{y})$ are the displacements function. We pose as boundary conditions 50 Pascal as shear stress on the beam (Neumann condition for $\mathrm{x}=21$ ) and $\mathbf{u}(10, \mathrm{y})=\mathbf{v}(10, \mathrm{y})=0$ as Dirichelet condition. These equations can be solved numerically; if we discretize our beam by a $10 \times 10$ square lattice; the solution is shown in Figure 27 and the Von Mises plot in Figure 28; deformed mesh are plotted in red colour.

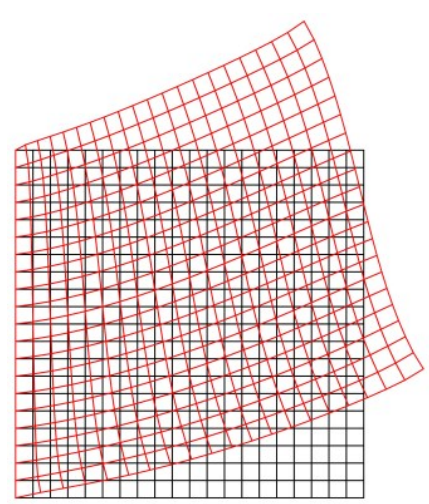

Figure 27 FEM solutions of bidimensional square beam under shear stress

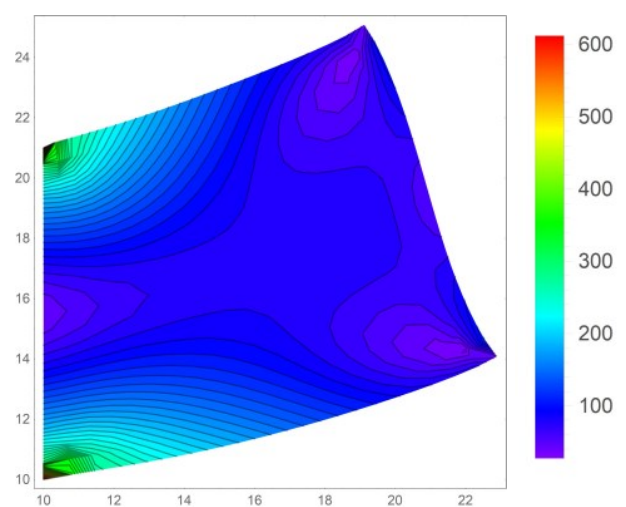

Figure $\mathbf{2 8}$ Von Mises plot of FEM solutions

To apply our tool we assign the displacements of some points, the leaders, make some choice about the algorithm (Lattice, interaction rules between the followers etc..) and compute the strain when the followers readjust themselves, after sufficiently long time. As leaders we have chosen the right and left side of the beam, so we assign the displacements of these points as obtained from the FEM equations and investigate the arrangement of the other points. The important thing to point out is that we still have no criteria about the choice of what lattice, interaction law between followers etc... So as first attempt we use a square lattice and no weight in the compute of the followers coordinates In Figure 29 the obtained configuration, together with the FEM solution (red points) are shown; in Figure 30 the corresponding Von Mises plot. The points on the left and on the right of the beam are overlapped because are the leaders and we have imposed their displacement as the FEM solution beam 
deformation. It can be outlined as external configuration of the beam is quite the same but the internal displacement of the points, i.e. the strain, is different; this can be highlighted if we compare the Von Mises plot of Figure 28 and Figure 30. Change in tool's parameters lead to different configuration, corresponding to different strain of the beam, as shown in a paper in press; almost none of them are satisfactory.

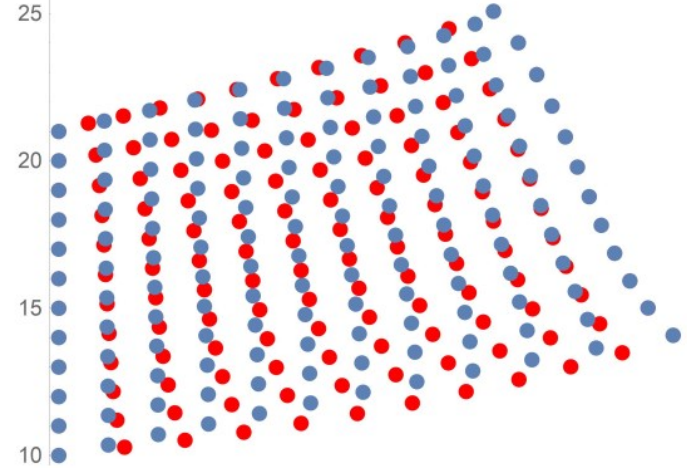

Figure 29 Beam deformation

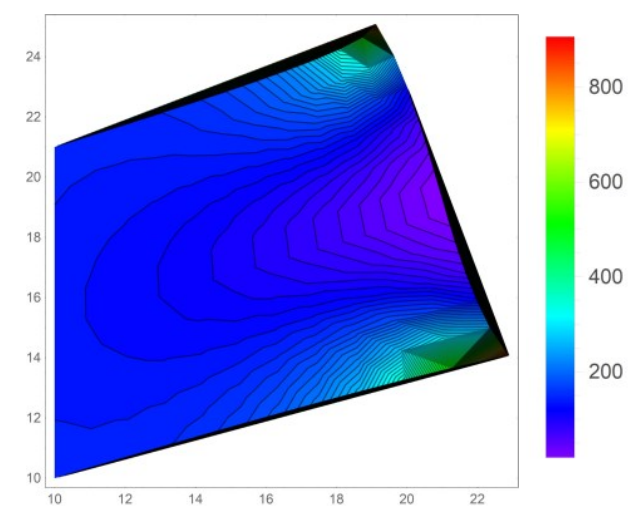

Figure 30 Corresponding Von Mises Plot

Second gradient case has showed no appreciable differences; we have tried to give a quantitative measurements of the of the discrepancies with the FEM solutions, using the average value of the sum of the square differences between coordinates, in many cases. Nobody emerges as the best match so we can conclude that the beam deformation can be sometimes very similar to the FEM solution but the Von Mises stress plot is quite always unsatisfactory. This point need to be study again to a better understanding of the physic behind the tool and what should be drive our choices in the tool to describe material continuum. We have to remember that the materials parameters $\mathrm{Y}$ and $v$ does not appear explicitly in our algorithm but they are hidden into the interaction relationship between the followers, the neighbours and the choice of the lattice. So far we have no idea on how to select our choices to match the well known problem. Changing the parameters of our tool we obtain different results nobody of them perfectly coincident with classical solution but, owing to the flexibility of the tool, we believe it exist a parameters combination to fit the deformed beam; but this is meaningless until we do not understand how to choose the parameters.. The reason of this lie in the fact that, up to now, we do not start from the constitutive equations of the materials leading to the rules governing points displacement. We have to work on how to connect the rules of our model with classical physical proprieties of the material.

\section{Case i) Long beam}

Also in the case of longer beam we can try many combination of the parameters tool to fit the deflection of our beam but it is useless: we need to connect the constitutive parameters with the tool. If we increase the ratio length/width of our beam from 1 to 5 the results are still different (see Figure 31,32,33 and 34)). As can be seen the results do not behave as we expected and show a wide range of possibilities in which we could choose. Similar results were obtained if we increase the ratio length/width up to 10 or more. Although the external shape of the beam can sometimes be acceptable, once again we can see as the displacement of the internal particles is quite different from the FEM solutions; it is clear that the differences are sometimes noticeable, as outlined by the Von Mises' graphs. So far we stop trial and errors to think about a better understanding of the physic behind the tool and what should drive our choices in the tool to describe material continuum. This will be the object of the next paper. 


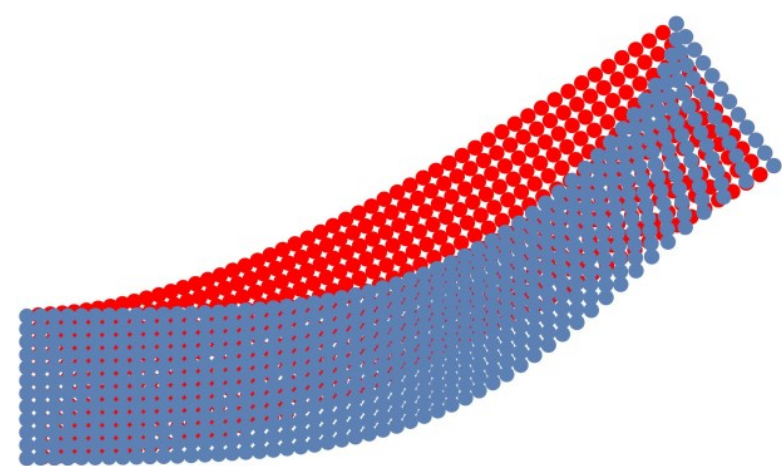

Figure 31 Square lattice

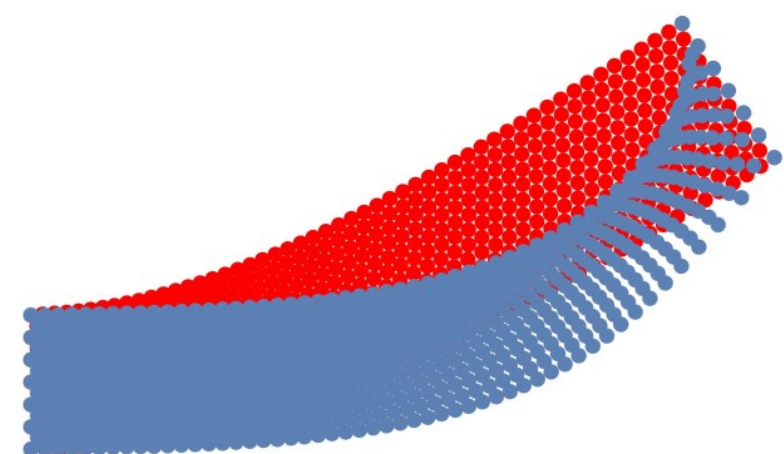

Figura 33 Hexagonal lattice

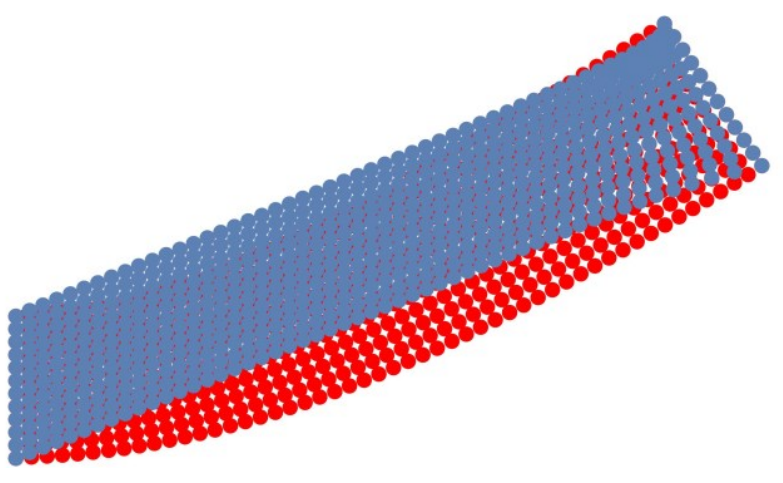

Figure 32 Square lattice reduced neighbours numbers

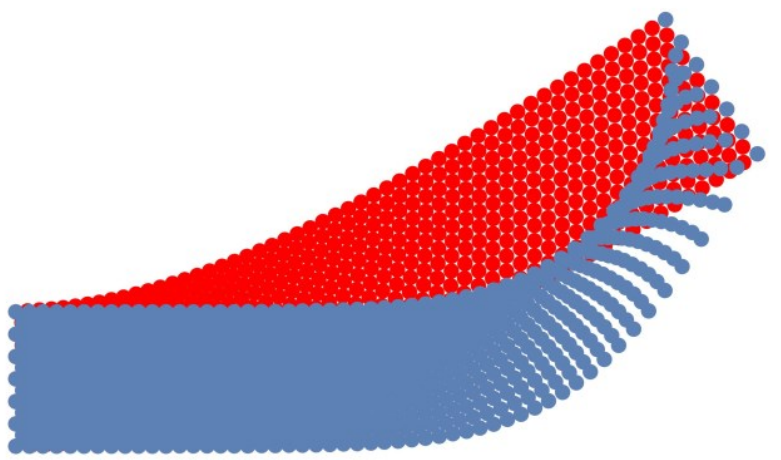

Figure 34 Hexagonal lattice reduced neighbours numbers

\section{Future work and conclusion}

The most important topic is to investigate how to relate materials parameters with the choices we are doing in our tool, to achieve a connection with the usual methods of Continuum Mechanic. Actually we have not criteria about how direct our tool's choices to describe a particular material continuum. In the beam deformation we have used Young modulus and Poisson coefficient assigned but there is no relationship with the parameters of our model. This is the reason of the discrepancy in the resulting deformation compared to FEM solution.

Generalization in 3D of the tool is quite easy but still needs some optimization in the code to keep the computation time in the order of seconds, by using a normal PC Desktop. Moreover we are relaxing the hypothesis that the neighbours always are the same to describe liquid and gas; this needs a calculation step more because you have to compute the neighbours, defined in this case as the particles inside a specified volume, at each time step. Another interesting feature we are introducing is constrained on the particle's motion to describe structured object like pantograph [51-58]. It can be described as a set of beams with constrained point in the pivot; in the Hencky vision can be conceived as a set of point interconnected by springs. Further developments are concerning different fracture mechanism, different frame to avoid edge effects, other interactions rules and adaptive lattice. Cellular automata seems to be a good candidate to enhance our work; A cellular automata is a simple computational mechanism that, for example, changes the colour of each cell on a lattice based on the colour of neighbours cells according to a transformation rule. Some attempts to use them in Mechanics have been done [59-60]. Principal limit of Cellular automata is regarding as do not evolve sufficiently, so they quickly reach a limited asymptote in their order of complexity and this will be the object of a future paper.

Working on flocking rules, governing the configuration of an underwater robot swarm, we have found they can profitably used to describe deformation of bi-dimensional continuum medium. In this work we have discussed about a tool, presented in previous works, able to describe strain deformation of a continuum medium taking in account complex physical effects in a plausible way. The tool is based on Position Based Dynamic. Differently from the PBD methods used in computer graphics, we still do not ask for the knowledge of the velocity and do not introduce some kind of forces to take in account mechanical effects. The strain is imposed on some particles (leaders) whose motion is assigned and the other particles (followers) move according to some rules governing particle position. The motion of the followers is determined, like in a bird swarm, by the position of their 
neighbours. So far the deformed configuration is calculated not by Newton law but only by the relative positions between the particles of the system, the characteristics of the lattice and by rules describing the how a particle would like to place with respect to its neighbours. One of the principal advantages is in the saving machine time for computing. Essentially we have to compute the action of a transformation operator between matrices and the job can be parallelized between the GPU cores of the powerful video card, saving computational cost need to solve FEMs. The presented results are interesting but they still are at a preliminary stage. Edge effects are take in account by a frame and fracture mechanism is described by a threshold effect. We have showed as changing some parameters like, lattice, interaction rules, fracture distance, numbers of neighbours much different behaviour can be described. Pseudo energetic considerations are introduced to describe different deformation regimes, such as elastic and plastic and to achieve a better understanding of the process. This is preliminary to introducing potential descriptive interactions depending on the relative distance between the particles, which are able to reproduce the well known physical behaviour. The tool we propose could be considered as just a graphic representation of a plausible behaviour because, actually, we imitate a known behaviour adjusting the algorithm parameters. In previous works we showed as results of this tool has good similarity with the predictions of standard FEM simulations, also in fracture case. Working with a transformation operator between matrices the job can be parallelized between the GPU cores of the powerful video card, saving computational cost usually associated with FEMs. The presented results are interesting but they still are at a preliminary stage. In previous works we showed as results of this tool has, in some cases, good similarity with the predictions of standard FEM simulations, also in fracture case. In this paper we outlined also some discrepancies with respect to FEM solution like for a beam under shear stress. We have collected some success showing plausible deformation in different conditions but when we consider a beam under loading the need to connect constitutive equations with the parameters of our tool emerges powerfully. However the tool has demonstrated enough flexibility to give chances that, once connected with the constitutive parameters, it can describe the richness of behaviour of different materials, including potentially complex biological tissues.

\section{Acknowledgments}

The author is grateful to Prof. Victor A. Eremeyev, Prof Pierre Seppecher and Prof. Francesco Dell'Isola for the fruitful discussion on this topic we had in MEMOCS Workshop on Models of Complex Materials and Systems last June in Arpino.

\section{Funding}

The authors received no financial support for the research, authorship, and/or publication of this article.

\section{Bibliography}

[1] J. Bender, M. Müller, e M. Macklin, «Position-Based Simulation Methods in Computer Graphics.», in Eurographics (Tutorials), 2015 [Online]. Available at: https://www.researchgate.net/profile/Jan_Bender/publication/274940214_PositionBased Simulation_Methods in Computer_Graphics/links/552cc4a40cf29 b22c9c466df/PositionBased-Simulation-Methods-in-Computer-Graphics.pdf. [Consultato: 06-set-2017]

[2] N. Umetani, R. Schmidt, e J. Stam, «Position-based elastic rods», in Proceedings of the ACM SIGGRAPH/Eurographics Symposium on Computer Animation, 2014, pagg. 21-30.

[3] R. dell'Erba, «Determination of Spatial Configuration of an Underwater Swarm with Minimum Data», Int. J. Adv. Robot. Syst., vol. 12, n. 7, pag. 97, lug. 2015.

[4] C. Moriconi e R. dell'Erba, «The Localization Problem for Harness: A Multipurpose Robotic Swarm», in SENSORCOMM 2012, The Sixth International Conference on Sensor Technologies and Applications, 2012, pagg. 327-333 [Online]. Available at: $\mathrm{http} / / /$ www.thinkmind.org/index.php?view=article\&articleid=sensorcomm_2012_14_20_10138. [Consultato: 04-apr-2014] 
[5] A. Battista, L. Rosa, R. dell’Erba, e L. Greco, «Numerical investigation of a particle system compared with first and second gradient continua: Deformation and fracture phenomena*», Math. Mech. Solids, pag. 1081286516657889, lug. 2016.

[6] R. dell'Erba, «Position-based dynamic of a particle system: a configurable algorithm to describe complex behaviour of continuum material starting from swarm robotics», Contin. Mech.

Thermodyn., pagg. 1-22, 2018.

[7] R. dell'Erba, «Swarm robotics and complex behaviour of continuum material», Contin. Mech. Thermodyn., pagg. 1-26, 2018.

[8] H. Seddik, R. Greve, L. Placidi, I. Hamann, e O. Gagliardini, «Application of a continuummechanical model for the flow of anisotropic polar ice to the EDML core, Antarctica», J. Glaciol., vol. 54, n. 187, pagg. 631-642, 2008.

[9] L. Placidi, R. Greve, H. Seddik, e S. H. Faria, «Continuum-mechanical, Anisotropic Flow model for polar ice masses, based on an anisotropic Flow Enhancement factor», Contin. Mech. Thermodyn., vol. 22, n. 3, pagg. 221-237, 2010.

[10] W. Pietraszkiewicz e V. A. Eremeyev, «On natural strain measures of the non-linear micropolar continuum», Int. J. Solids Struct., vol. 46, n. 3, pagg. 774-787, 2009.

[11] J. Altenbach, H. Altenbach, e V. A. Eremeyev, «On generalized Cosserat-type theories of plates and shells: a short review and bibliography», Arch. Appl. Mech., vol. 80, n. 1, pagg. 73-92, 2010.

[12] V. A. Eremeyev, L. P. Lebedev, e H. Altenbach, Foundations of micropolar mechanics. Springer Science \& Business Media, 2012.

[13] H. Altenbach, V. A. Eremeyev, L. P. Lebedev, e L. A. Rendón, «Acceleration waves and ellipticity in thermoelastic micropolar media», Arch. Appl. Mech., vol. 80, n. 3, pagg. 217-227, 2010.

[14] H. Altenbach e V. A. Eremeyev, Generalized Continua From the Theory to Engineering Applications, CISM Courses and Lectures, vol. 541. Springer, Udine, 2013.

[15] Barchiesi, E., dell'Isola, F., Laudato, M., Placidi, L., \& Seppecher, P. (2018). A 1D Continuum Model for Beams with Pantographic Microstructure: Asymptotic Micro-Macro Identification and Numerical Results. In Advances in Mechanics of Microstructured Media and Structures (pp. 43-74). Springer, Cham.

[16] Placidi, L., Rosi, G., \& Barchiesi, E. (2019). Analytical Solutions of 2-dimensional Second Gradient Linear Elasticity for Continua with Cubic-D 4 Microstructure. In New Achievements in Continuum Mechanics and Thermodynamics (pp. 383-401). Springer, Cham.

[17] Rosi, G., Placidi, L., \& dell'Isola, F. (2017). "Fast" and "slow" pressure waves electrically induced by nonlinear coupling in Biot-type porous medium saturated by a nematic liquid crystal. Zeitschrift für angewandte Mathematik und Physik, 68(2), 51.

[18] Turco, E. (2019). How the Properties of Pantographic Elementary Lattices Determine the Properties of Pantographic Metamaterials. In New Achievements in Continuum Mechanics and Thermodynamics (pp. 489-506). Springer, Cham.

[19] Turco, E., Golaszewski, M., Giorgio, I., \& Placidi, L. (2017). Can a Hencky-Type Model Predict the Mechanical Behaviour of Pantographic Lattices?. In Mathematical Modelling in Solid Mechanics (pp. 285-311). Springer, Singapore.

[20] Franciosi, P., \& Lebail, H. (2004). Anisotropy features of phase and particle spatial pair distributions in various matrix/inclusions structures. Acta materialia, 52(10), 3161-3172.[26] P. Franciosi, "A regularized multi-laminate-like plasticity scheme for polycrystals, applied to the FCC structure," Procedia IUTAM, vol. 3, pp. 141-156, 2012.

[21] B. E. Abali, W. H. Müller, e F. Dell'Isola, «Theory and computation of higher gradient elasticity theories based on action principles», Arch. Appl. Mech., vol. 87, n. 9, pagg. 1495-1510, 2017. 
[22] M. Cuomo, F. dell'Isola, L. Greco, e N. L. Rizzi, «First versus second gradient energies for planar sheets with two families of inextensible fibres: investigation on deformation boundary layers, discontinuities and geometrical instabilities», Compos. Part B Eng., vol. 115, pagg. 423-448, 2017.

[23] E. Turco, F. dell'Isola, A. Cazzani, e N. L. Rizzi, «Hencky-type discrete model for pantographic structures: numerical comparison with second gradient continuum models», Z. Für Angew. Math. Phys., vol. 67, n. 4, pag. 85, 2016.

[24] F. dell'Isola, A. Madeo, e P. Seppecher, «Cauchy tetrahedron argument applied to higher contact interactions», Arch. Ration. Mech. Anal., vol. 219, n. 3, pagg. 1305-1341, 2016.

[25] F. dell'Isola, P. Seppecher, e A. D. Corte, «The postulations á la D'Alembert and á la Cauchy for higher gradient continuum theories are equivalent: a review of existing results», Proc. R. Soc. Math. Phys. Eng. Sci., vol. 471, n. 2183, pag. 20150415, 2015.

[26] A. Javili, F. dell'Isola, e P. Steinmann, «Geometrically nonlinear higher-gradient elasticity with energetic boundaries», J. Mech. Phys. Solids, vol. 61, n. 12, pagg. 2381-2401, 2013.

[27] P. Seppecher, J.-J. Alibert, e F. D. Isola, «Linear elastic trusses leading to continua with exotic mechanical interactions», in Journal of Physics: Conference Series, 2011, vol. 319, pag. 012018.

[28] S. Forest, N. M. Cordero, e E. P. Busso, «First vs. second gradient of strain theory for capillarity effects in an elastic fluid at small length scales», Comput. Mater. Sci., vol. 50, n. 4, pagg. 12991304, 2011.

[29] L. Placidi, «A variational approach for a nonlinear 1-dimensional second gradient continuum damage model», Contin. Mech. Thermodyn., vol. 27, n. 4-5, pagg. 623-638, 2015.

[30] Andreaus, U., dell'Isola, F., Giorgio, I., Placidi, L., Lekszycki, T., \& Rizzi, N. L. (2016). Numerical simulations of classical problems in two-dimensional (non) linear second gradient elasticity. International Journal of Engineering Science, 108, 34-50.

[31] Abali, B. E., Müller, W. H., \& Eremeyev, V. A. (2015). Strain gradient elasticity with geometric nonlinearities and its computational evaluation. Mechanics of Advanced Materials and Modern Processes, 1(1), 4.

[32] Placidi, L., Greco, L., Bucci, S., Turco, E., \& Rizzi, N. L. (2016). A second gradient formulation for a 2D fabric sheet with inextensible fibres. Zeitschrift für angewandte Mathematik und Physik, 67(5), 114.

[33] L. Placidi, I. Giorgio, A. Della Corte, e D. Scerrato, «Euromech 563 Cisterna di Latina 17-21 March 2014 Generalized continua and their applications to the design of composites and metamaterials: a review of presentations and discussions», Math. Mech. Solids, vol. 22, n. 2, pagg. 144-157, 2017.

[34] F. dell'Isola, D. Steigmann, e A. Della Corte, «Synthesis of fibrous complex structures: designing microstructure to deliver targeted macroscale response», Appl. Mech. Rev., vol. 67, n. 6, pag. 060804, 2015.

[35] T. Bückmann et al., «Tailored 3D mechanical metamaterials made by dip-in direct-laser-writing optical lithography», Adv. Mater., vol. 24, n. 20, pagg. 2710-2714, 2012.

[36] dell'Isola, F., Seppecher, P., Alibert, J. J., Lekszycki, T., Grygoruk, R., Pawlikowski, M., ... \& Gołaszewski, M. (2018). Pantographic metamaterials: an example of mathematically driven design and of its technological challenges. Continuum Mechanics and Thermodynamics, 1-34.

[37] Barchiesi, E., Spagnuolo, M., \& Placidi, L. (2019). Mechanical metamaterials: a state of the art. Mathematics and Mechanics of Solids, 24(1), 212-234.

[38] Carcaterra, A., dell'Isola, F., Esposito, R., \& Pulvirenti, M. (2015). Macroscopic description of microscopically strongly inhomogenous systems: A mathematical basis for the synthesis of higher gradients metamaterials. Archive for Rational Mechanics and Analysis, 218(3), 1239-1262.

[39] Turco, E., Giorgio, I., Misra, A., \& Dell'Isola, F. (2017). King post truss as a motif for internal structure of (meta) material with controlled elastic properties. Royal Society open science, 4(10),

171153 . 
[40] dell'Isola, F., Bucci, S., \& Battista, A. (2016). Against the fragmentation of knowledge: The power of multidisciplinary research for the design of metamaterials. In Advanced Methods of

Continuum Mechanics for Materials and Structures (pp. 523-545). Springer, Singapore.[41]

Milton, G., \& Seppecher, P. (2012). A metamaterial having a frequency dependent elasticity tensor and a zero effective mass density. physica status solidi (b), 249(7), 1412-1414.

[42] C. Lanczos, The variational principles of mechanics. Courier Corporation, 2012.

[43] L. Placidi, F. dell'Isola, N. Ianiro, e G. Sciarra, «Variational formulation of pre-stressed solidfluid mixture theory, with an application to wave phenomena», Eur. J. Mech. ASolids, vol. 27, n. 4, pagg. 582-606, 2008.

[44] F. dell'Isola e L. Placidi, «Variational principles are a powerful tool also for formulating field theories», in Variational models and methods in solid and fluid mechanics, Springer, 2011, pagg. 115.

[45] F. dell'Isola, A. Della Corte, L. Greco, e A. Luongo, «Plane bias extension test for a continuum with two inextensible families of fibers: a variational treatment with Lagrange multipliers and a perturbation solution», Int. J. Solids Struct., vol. 81, pagg. 1-12, 2016.

[46] F. dell'Isola e S. Gavrilyuk, Variational models and methods in solid and fluid mechanics, vol. 535. Springer Science \& Business Media, 2012.

[47] F. dell'Isola, N. Auffray, V. A. Eremeyev, A. Madeo, L. Placidi, e G. Rosi, «Least action principle for second gradient continua and capillary fluids: A Lagrangian approach following Piola's point of view», in The complete works of Gabrio Piola: Volume I, Springer, 2014, pagg. 606-694.

[48] M. Avella, R. DelL'Erba, e E. Martuscelli, «Fiber reinforced polypropylene: Influence of iPP molecular weight on morphology, crystallization, and thermal and mechanical properties», Polym. Compos., vol. 17, n. 2, pagg. 288-299, 1996.

[49] M. Avella, R. dell'Erba, L. D’Orazio, e E. Martuscelli, «Influence of molecular weight and molecular weight distribution on crystallization and thermal behavior of isotactic polypropylene», Polym. Netw. Blends, vol. 5, n. 1, pagg. 47-54, 1995.

[50] M. Avella, R. dell'Erba, E. Martuscelli, e G. Ragosta, «Influence of molecular mass, thermal treatment and nucleating agent on structure and fracture toughness of isotactic polypropylene», Polymer, vol. 34, n. 14, pagg. 2951-2960, 1993.

[51] I. Giorgio, A. Della Corte, F. dell'Isola, e D. J. Steigmann, «Buckling modes in pantographic lattices», Comptes Rendus Mécanique, vol. 344, n. 7, pagg. 487-501, lug. 2016.

[52] Boutin, C., Giorgio, I., \& Placidi, L. (2017). Linear pantographic sheets: Asymptotic micro-macro models identification. Mathematics and Mechanics of Complex Systems, 5(2), 127-162.

[53] Giorgio, I., Harrison, P., dell'Isola, F., Alsayednoor, J., \& Turco, E. (2018). Wrinkling in engineering fabrics: a comparison between two different comprehensive modelling approaches. Proceedings of the Royal Society A: Mathematical, Physical and Engineering Sciences, 474(2216), 20180063.

[54] Spagnuolo, M., Barcz, K., Pfaff, A., Dell'Isola, F., \& Franciosi, P. (2017). Qualitative pivot damage analysis in aluminum printed pantographic sheets: numerics and experiments. Mechanics Research Communications, 83, 47-52.

[55] De Angelo, M., Spagnuolo, M., D’Annibale, F., Pfaff, A., Hoschke, K., Misra, A., ... \& Pawlikowski, M. (2019). The macroscopic behavior of pantographic sheets depends mainly on their microstructure: experimental evidence and qualitative analysis of damage in metallic specimens. Continuum Mechanics and Thermodynamics, 1-23. 
[56] Andreaus, U., Spagnuolo, M., Lekszycki, T., \& Eugster, S. R. (2018). A Ritz approach for the static analysis of planar pantographic structures modeled with nonlinear Euler-Bernoulli beams. Continuum Mechanics and Thermodynamics, 30, 1103-1123.

[57] Turco, E., Barcz, K., Pawlikowski, M., \& Rizzi, N. L. (2016). Non-standard coupled extensional and bending bias tests for planar pantographic lattices. Part I: numerical simulations. Zeitschrift für angewandte Mathematik und Physik, 67(5), 122.

[58] Turco, E., \& Rizzi, N. L. (2016). Pantographic structures presenting statistically distributed defects: numerical investigations of the effects on deformation fields. Mechanics Research Communications, 77, 65-69.

[59] Y. Dong, G. Zhang, A. Xu, e Y. Gan, «Cellular automata model for elastic solid material», Commun. Theor. Phys., vol. 59, n. 1, pagg. 59-67, gen. 2013.

[60] I. S. Konovalenko, A. Y. Smolin, e S. G. Psakhie, «Multilevel simulation of deformation and fracture of brittle porous materials in the method of movable cellular automata», Phys. Mesomech., vol. 13, n. 1-2, pagg. 47-53, gen. 2010. 